\title{
FINITE GROUPS VIEWED LOCALLY
}

\author{
BY J. L. ALPERIN ${ }^{1}$
}

1. Introduction. Groups have now been studied for over two hundred years. Their first great success, over one hundred and forty years ago, was Galois theory which exploited the relationship between polynomial equations and finite groups. This idea motivated further developments. For example, Lie groups were introduced to study differential equations in an analogous way.

A common theme in many parts of group theory has been the classification of simple groups. This was achieved for complex Lie groups by Killing and for real Lie groups by Cartan. Similar results for algebraic groups were obtained in work led by Chevalley. Only now does it seem likely that a classification can be accomplished for finite simple groups. This is a more complicated and difficult problem by far than any of the other classifications. The important applications of our present knowledge of finite simple groups will be followed by many more such results.

Apart from the basic ideas about normal structure, in particular, normal subgroups, homomorphisms, quotient groups, and direct products, there are three separate but intertwined methods of studying the structure of finite groups. First, we have the oldest: permutation groups. One studies the $G$-sets for a group $G$, the sets that $G$ acts on. This leads to all sorts of geometrical and combinatorial considerations. Second, there is the method of representation theory where one deals with $G$-modules, namely vector spaces, abelian groups and modules on which the group $G$ acts. Here one gets involved with characters and then algebras, number fields, division algebras, non-semisimple rings and homological algebra. Third, there is the local method, that part of the subject that begins with the Sylow theorems.

And this is our subject. We shall have a glimpse and a survey of the local method emphasizing the results that are so useful in studying simple groups. We shall not explore the other methods and we shall only touch on the vast web of relationships between these different methods. However, it is the way that these disparate methods so perfectly complement each other which is the basis for the tremendous progress on classifying finite simple groups.

We now come to the basic definitions. If $G$ is a finite group and $p$ is a prime then a subgroup of $G$ of order a power of $p$ is called a $p$-subgroup of $G$. If $Q$ is a nonidentity $p$-subgroup of $G$ then the normalizer $N(Q)$ is called a $p$-local subgroup of $G$, or simply a local subgroup of $G$. It is the $p$-local

An expanded version of an address to the Society given on November 1, 1975 in Chicago; received by the editors February 25, 1977.

${ }^{1}$ Supported in part by National Foundation Grant NSF GP-37575X.

c American Mathematical Society 1977 
subgroups rather than the $p$-subgroups which are important. Their structure and their relationships with $G$ are our subject here.

Let's look at an example to see that these local subgroups include important classes of subgroups. The best example of a finite group is the general linear group over a finite field (and not the examples which are usually given). Thus, let $G=\mathrm{GL}(5, q)$, the group of all nonsingular five by five matrices over a field with $q$ elements, where $q$ is a power of the prime $p$. Let

$$
Q=\left\{\left(\begin{array}{lllll}
1 & 0 & 0 & * & * \\
0 & 1 & 0 & * & * \\
0 & 0 & 1 & * & * \\
0 & 0 & 0 & 1 & 0 \\
0 & 0 & 0 & 0 & 1
\end{array}\right)\right\}
$$

the set of all matrices obtained by letting each star vary over the field. It follows that $Q$ is a subgroup and that it has order $q^{6}$. In particular, $Q$ is a nonidentity $p$-subgroup of $G$. It is easy to determine the normalizer of $Q$ and we get

$$
N(Q)=\left\{\left(\begin{array}{ccccc}
a_{11} & a_{12} & a_{13} & * & * \\
a_{21} & a_{22} & a_{23} & * & * \\
a_{31} & a_{32} & a_{33} & * & * \\
0 & 0 & 0 & b_{11} & b_{12} \\
0 & 0 & 0 & b_{21} & b_{22}
\end{array}\right)\right\}
$$

Moreover, there is an exact sequence

$$
1 \rightarrow Q \rightarrow N(Q) \rightarrow \mathrm{GL}(3, q) \times \mathrm{GL}(2, q) \rightarrow \mathrm{I}
$$

where the last maps sends

$$
\left(\begin{array}{ll}
A & * \\
0 & B
\end{array}\right)
$$

to the ordered pair $(A, B)$. Thus, this $p$-local subgroup is one of the so-called parabolic subgroups of $G$. In fact, all of the parabolic subgroups are $p$-local subgroups.

But what about local subgroup for primes other than the characteristic? Can they be interesting? Suppose that $p$ is odd and let $t$ be an involution of $G$, that is, an element of order two. Thus, $t^{2}=1$ so the minimum polynomial of the matrix $t$ divides $X^{2}-1$. Since this polynomial has the two distinct roots +1 and -1 , it follows that $t$ is similar to a diagonal matrix with diagonal entries each +1 or -1 . That is, $t$ is conjugate in $G$ to such a matrix. Thus, a typical involution would be the matrix 


$$
t=\left(\begin{array}{rrrrr}
-1 & 0 & 0 & 0 & 0 \\
0 & -1 & 0 & 0 & 0 \\
0 & 0 & -1 & 0 & 0 \\
0 & 0 & 0 & 1 & 0 \\
0 & 0 & 0 & 0 & 1
\end{array}\right)
$$

The centralizer $C(t)$ of $t$ is a 2-local subgroup, being the normalizer of the group of order two consisting of $t$ and 1 . And this centralizer is easy to determine:

$$
C(t)=\left\{\left(\begin{array}{lllll}
* & * & * & 0 & 0 \\
* & * & * & 0 & 0 \\
* & * & * & 0 & 0 \\
0 & 0 & 0 & * & * \\
0 & 0 & 0 & * & *
\end{array}\right)\right\}
$$

which is one of the Levi complements.

The theory of local subgroups now divides into three parts, which deal with the following three situations: a single $p$-local subgroup; the collection of all $p$-local subgroups; the relations between the $p$-local subgroups and the $q$-local subgroups for distinct primes $p$ and $q$. We shall discuss each of these topics in turn and then examine the fundamental " $B$-conjecture" and make some concluding remarks.

2. One $p$-local subgroup. Let $Q$ be a nonidentity $p$-subgroup of the group $G$ and let $L=N(Q)$ be the corresponding $p$-local subgroup of $G$. For the sake of simplicity let's assume as well that $Q$ is abelian and of exponent $p$, so that $x^{p}=1$ for every $x$ in $Q$. We can now give $Q$ the structure of a vector space over the field $F_{p}$ of integers modulo $p$. If $x$ and $y$ are in $Q$ we set $x+y=x y$ while if $f$ is in $F_{p}$ then $f x=x^{n}$ where $n$ is any integer in the residue class $f$. All the required properties are easily verified. Moreover, the group $L$ acts on $Q$. If $x$ is in $Q$ and $h$ is in $L$ then set $x \cdot h=h^{-1} x h$. It follows that if $h$ and $k$ are in $L$ then $f(x \cdot h)=f x \cdot h, x h \cdot k=x \cdot h k$ and $Q$ has the structure of an $F_{p} L$ module, where $F_{p} L$ is the group algebra of $L$ over $F_{p}$.

For example, if $G$ is now the example of the previous section and we take

$$
h=\left(\begin{array}{ll}
A & 0 \\
0 & B
\end{array}\right), \quad x=\left(\begin{array}{ll}
I & X \\
0 & I
\end{array}\right)
$$

then

$$
x \cdot h=\left(\begin{array}{cc}
I & A^{-1} X B \\
0 & I
\end{array}\right)
$$

In general, one can now use all sorts of module techniques to analyze the structure of $L$. We can extend the field $F_{p}$ to an algebraically closed field, look at eigenvalues of linear transformations, employ homological techniques and do many other things. 
If $Q$ is not abelian of exponent $p$ then these ideas can still be used. In fact, if $R$ and $S$ are normal subgroups of $L$ with $Q \geqslant R \geqslant S$ and $R / S$ abelian of exponent $p$ then $R / S$ becomes an $F_{p}[L / S]$ module. Moreover, one can make many choices of such modules and their structures are related. This brings in considerations from multilinear algebra and the use of tensor products and exterior algebras.

One very important topic in the theory of a single $p$-local subgroup is that of factorizations. The idea is to express $L$ as the product of certain of its subgroups, namely, the largest normal subgroup of $L$ of order not divisible by $p$ and the normalizers of appropriate characteristic subgroups of a Sylow $p$-subgroup of $L$. The main use of these factorizations is to establish that $L$ contains a Sylow $p$-subgroup of $G$ when $L$ is a maximal $p$-local subgroup.

At this point we hope the reader has a picture of how to look at a $p$-local subgroup of a group. We're now going to explore how all these local subgroups are related and what they tell us about the group in which they are contained.

3. All the $p$-local subgroups. There are really two separate problems here. Either we know one or several $p$-local subgroups of a group $G$ and we want to know them all or we do indeed know them all and we want to deduce global information, that is, information about $G$. We will describe these two problems in turn, emphasizing the latter one.

Suppose that $Q$ is a nonidentity $p$-subgroup of the group $G$, that $L=$ $N(Q)$ and that we have a good grasp on $L$. Now suppose that $R$ is another nonidentity $p$-subgroup of $G$. What can we say about $M=N(R)$ ? If $R$ centralizes $Q$ so that $Q$ is in $L$ then we can say quite a bit. Moreover, by iterating this argument we can in fact get at all the $p$-local subgroups of $G$.

To explain this simple idea let's have a picture:

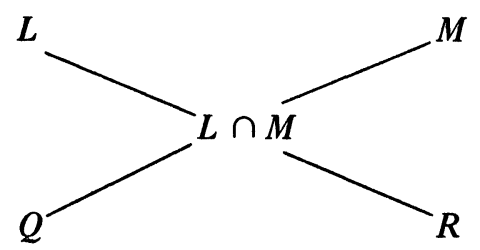

We are given $L, Q$ and $R$ with $Q$ and $R$ commuting elementwise. Thus, $L \cap M$ consists of the elements of $L$ which normalize $R$ so $L \cap M$ is determined. But this intersection is also the set of elements of $M$ which normalize $Q$. That is, in the unknown group $M$ we have $Q$ given and also $N_{M}(Q)$. Now usually $L \cap M$ is smaller than $L$. Hence, $M$ is a group in which we know a $p$-local subgroup and presumably by induction we can determine the other $p$-local subgroups of $M$ and get at $M$ itself. This is really an art and not a science and requires a lot of patience and ingenuity. There are thousands of pages of journals devoted to just this problem in endless variations. 
Now let's turn to the second problem. Let $P$ be a Sylow $p$-subgroup of the group $G$ and suppose that for any nonidentity subgroup $R$ of $P$ we have $N_{G}(R)$ so that, in a manner of speaking, we have the complete $p$-local structure of $G$. We wish to get at $G$ itself. Here's a picture:

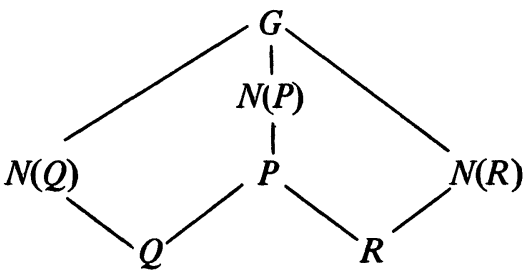

One of the important things to know about $G$ is its conjugacy classes. Therefore, if $x$ and $y$ are elements of $P$ we would like to know whether or not $x$ and $y$ are conjugate in $G$. It can certainly happen that they are without being conjugate in $P$. For example, the elements $x$ and $y$ could be locally conjugate, that is, contained in a nonidentity subgroup $S$ of $P$ and conjugate in $N_{G}(S)$.

Let's have a look at an example. Let $G=\mathrm{GL}(3, p)$ and let

$$
P=\left\{\left(\begin{array}{lll}
1 & * & * \\
0 & 1 & * \\
0 & 0 & 1
\end{array}\right)\right\}
$$

so $P$ has order $p^{3}$ and is a Sylow $p$-subgroup of $G$. Set

$$
x=\left(\begin{array}{lll}
1 & 1 & 0 \\
0 & 1 & 0 \\
0 & 0 & 1
\end{array}\right), \quad y=\left(\begin{array}{lll}
1 & 0 & 0 \\
0 & 1 & 1 \\
0 & 0 & 1
\end{array}\right)
$$

so that $x$ and $y$ are conjugate in $G$ being similar matrices-they are both in Jordan canonical form. They are not in fact even locally conjugate. But, if we set

$$
\begin{gathered}
z=\left(\begin{array}{lll}
1 & 0 & 1 \\
0 & 1 & 0 \\
0 & 0 & 1
\end{array}\right), \\
Q=\left\{\left(\begin{array}{lll}
1 & * & * \\
0 & 1 & 0 \\
0 & 0 & 1
\end{array}\right)\right\}, \quad R=\left\{\left(\begin{array}{lll}
1 & 0 & * \\
0 & 1 & * \\
0 & 0 & 1
\end{array}\right)\right\}
\end{gathered}
$$

then $x$ and $z$ are in $Q$ and conjugate in $N(Q)$ while $z$ and $y$ are in $R$ and are conjugate in $N(R)$.

Thus, the elements $x$ and $y$ are conjugate and this can be seen by a sequence of local conjugations. This is typical as the fusion theorem shows: If $x$ and $y$ are elements of the Sylow p-subgroup $P$ of the group $G$ and $x$ and $y$ are 
conjugate in $G$ then there are elements $x_{1}, \ldots, x_{n}$ of $P$ with $x=x_{1}, y=x_{n}$ and each pair of elements $x_{i}, x_{i+1}$ being locally conjugate. Actually, there is a lot more information most of which is necessary for applications. We have $x_{i}, x_{i+1}$ in a subgroup $Q_{i}$ of $P$ with $t_{i}^{-1} x_{i} t_{i}=x_{i+1}$ for some $t_{i}$ in $N\left(Q_{i}\right)$. We have to know which subgroups $Q_{i}$ actually arise as well as what elements $t_{i}$ are used. In fact, one can vary these a lot and tailor them to the particular problem at hand. But without going into these details let's discuss a few ways this can be used.

There are a lot of results of a sort of local-global nature which are very formal sounding. Let's establish the oldest of these-a result of Frobenius from the nineteenth century-by these methods. A group $G$ is called $p$-nilpotent if there is a homomorphism of $G$ onto a Sylow $p$-subgroup $P$. This is a very strong property certainly guaranteeing that $G$ is not simple! This is equivalent, by elementary arguments from representation theory, to the following: two elements of $P$ are conjugate in $G$ if, and only if, they are conjugate in $P$. Frobenius proved the following: The group $G$ is p-nilpotent if, and only if, every p-local subgroup of $G$ is p-nilpotent.

The proof is direct except for the demonstration that if every $p$-local subgroup of $G$ is $p$-nilpotent then any two elements of $P$ conjugate in $G$ are already conjugate in $P$. However, in view of the fusion theorem it suffices to show the following: if $x$ and $y$ are elements of $P$ contained in the nonidentity subgroup $Q$ of $P$ and conjugate in $N(Q)$ then $x$ and $y$ are conjugate in $P$. Using one of the details of the fusion theorem that we didn't mention one can also assume that $N_{P}(Q)=N(Q) \cap P$ is a Sylow $p$-subgroup of $N(Q)$. Here's the picture:

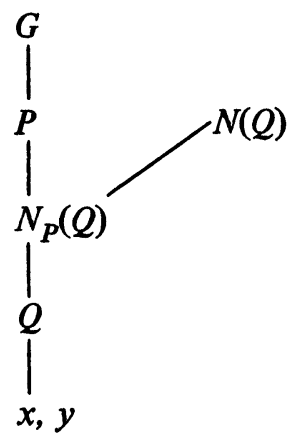

But $x$ and $y$ are elements of the Sylow $p$-subgroup $N_{p}(Q)$ of $N(Q)$ and $N(Q)$ is $p$-nilpotent so $x$ and $y$, being conjugate in $N(Q)$, are already conjugate in $N_{P}(Q)$. Since $N_{P}(Q)$ is a subgroup of $P$ it follows that $x$ and $y$ are conjugate in $P$ and the theorem is proved.

We can apply these methods to a sort of dual problem as well, that is, to get $p$-subgroups as the kernels of homomorphisms instead of as the images of homomorphisms. This is another special case of general local-global results. Keeping our notation, the hypotheses of the next result basically say this: we 
can find normal $p$-subgroups locally in a formal sort of way. The conclusion says that we are near to doing the same for $G$.

Hence, we assume the following conditions:

1. For each nonidentity $p$-subgroup $Q, W(Q)$ is a nonidentity subgroup of $Q$

2. If $Q$ and $R$ are nonidentity $p$-subgroups of $G$ and $x^{-1} Q x=R$ for $x$ in $G$ then $x^{-1} W(Q) x=W(R)$;

3. If $Q$ is as above, $L=N(Q)$ and $R$ is a Sylow $p$-subgroup of $L$ then $W(R)$ is normal in $L$.

Now, what we would like is that $W(P)$ is normal in $G$, or something close to that. What is true is the following: Under the above hypotheses, no element of $W(P)$ is conjugate in $G$ to an element of $P$ not in $W(P)$.

How good is this? Much better than it looks. The property that $W(P)$ has makes it look like it is the intersection of a normal subgroup of $G$ with $P$. For if $N$ is a normal subgroup of $G$ then no element of $P \cap N$ is conjugate in $G$ to any element outside of $N$, in particular to no element of $P$ outside $P \cap N$, And in practice this property does usually mean that $W(P)$ is such an intersection.

The reader may also be wondering where we might hope to obtain such a function as $W$. This is related to the material in the preceding section: the study of factorizations is exactly where such functions arise!

Now let's turn to one final application of quite a different nature. A fundamental contribution to group theory by Brauer is a set of techniques which deal with groups of even order. These ideas are basically from permutation groups and representation theory but because the prime two is involved there is a mesh with local methods. In particular, Brauer in a paper with Fowler, developed formulae for the order of a group in terms of other information. Thompson has followed this line and proved a basic result called the Thompson order formula. We shall now put this result together with with fusion theorem.

Let $G$ be a group of even order with Sylow 2-subgroup $T$. Suppose that we have the 2-local structure of $G$. That is, for each nonidentity subgroup $S$ of $T$ we have $N(S)$. Therefore, we can read off whether or not two elements of $T$ are conjugate in $G$. We can thus tell whether or not $G$ has only one conjugacy class of involutions, that is, elements of order two. Using the Thompson order formula and the fusion theorem we have the following: If $G$ is a group of even order then the 2-local structure determines the order of $G$ unless, perhaps, $G$ has a single conjugacy class of involutions.

Putting all these ideas together, if we start with a single 2-local subgroup of a group we can use the above techniques to get at the complete 2-local structure of the group and then we usually have the order of the group as a consequence. We're then hopefully within reach of the structure of $G$ itself by more long analysis.

To conclude this section we're going to discuss a very fundamental prob- 
lem which pertains to the relationship between local methods and representation theory. Recall that a representation of the group $G$ is a homomorphism of $G$ into $\operatorname{GL}(n, C)$, a complex general linear group of some dimension. Its character is the complex valued function on $G$ which attaches to each group element the trace of matrix representing it. Information about the values of these functions is actually very basic to studying the structure of the group $G$ and is a part of the work on simple groups. One of the deepest parts of representation theory is block theory which uses representation theory over fields of prime characteristic to get at these character values. Many of the main results of this theory are partial answers to the basic problem: Give simple rules for determining the values of characters of $G$ on elements of order divisible by $p$ in terms of the p-local structure of $G$. There have been great successes in this direction; much more remains to be done. It is very interesting to note how we have here elements of order divisible by $p, p$-local subgroups and representations in characteristic $p$ all tied up together!

We now end this digression and return to our discussion of local methods.

4. Semilocal theory. Let's now bring in local subgroups for more than one prime. The basic idea is still very much the same. Let $P$ be a nonidentity $p$-subgroup and let $Q$ be a nonidentity $q$-subgroup of the group $G$ with $p$ and $q$ distinct primes. Let $L=N(P)$ and $M=N(Q)$ so these are $p$-local and $q$-local subgroups, respectively. Suppose that $P$ and $Q$ commute elementwise so we have as before the following diagram:

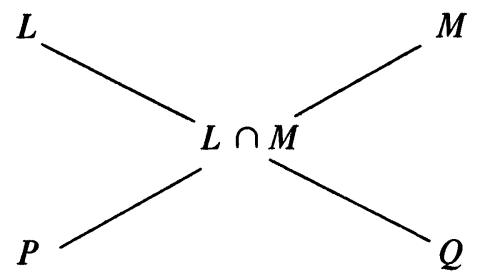

Again $L \cap M=N_{L}(Q)=N_{M}(P)$ so if we have a grasp on $L$ then we can work on $M$. We can start with one or more $p$-local subgroups and then get at a single $q$-local subgroup, explore all the $q$-local subgroups or go on to another prime $r$.

This is clearly a basic relationship being discussed here. The primes $p$ and $q$ should be considered related if there are subgroups $P$ and $Q$ as above. This will occur if, and only if, there is a subgroup of order $p$ and a subgroup of order $q$ which commute elementwise since $P$ and $Q$ have subgroups of such orders. This in turn occurs if, and only if, there is an element of order $p q$ in $G$. So we form a graph starting with the set of primes which divide the order of $G$ as the nodes and we form edges between two different nodes if there is an element of order the product of the two primes. Here are some examples (where $A_{13}$ is an alternating group and $\operatorname{SL}(4,4)$ is the group of four by four matrices of determinant one over a field of four elements): 


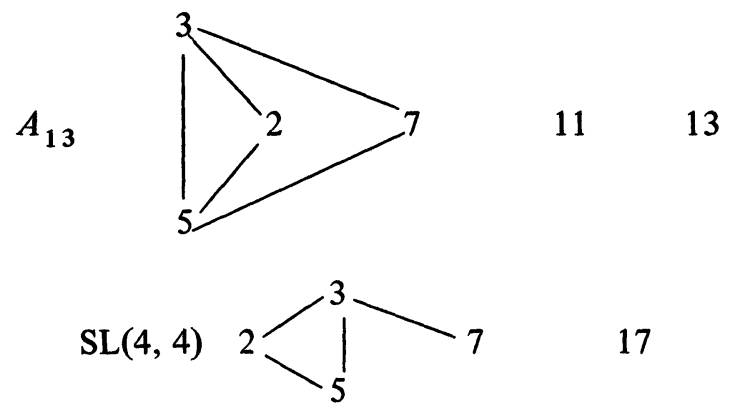

This gives some sort of idea of the sequences of arguments that can be used. Note also that there is more than one component in these examples; there are primes that you can't get at by starting with the prime two, which is an important point.

Now we shall focus our attention on a particular, but fundamental, problem of a semilocal nature. Let $G$ be a simple group and $t$ an involution of $G$. We would like to get at the 2-local subgroup $C(t)$, the centralizer of $t$. The place to start is actually $O(C(t))$, the largest normal subgroup of odd order in $C(t)$. Roughly, one would like that $O(C(t))=1$ or close to that. This is a very nontrivial question and also one whose solution gets one much farther towards unraveling the structure of simple groups then one would at first think. In much of the work on classifying simple groups if one can deal with $O(C(t))$ then one can actually get at $C(t)$. Having this one 2-local subgroup one can often determine the entire 2-local structure. This determines a lot about $G$, for example, usually its order, as we saw above, and certainly all the primes joined to the prime two in the graph we just constructed. Much more work can end up with the construction and classification of $G$ itself.

But we haven't really explained why this problem is of a semilocal nature. So let $G$ and $t$ be as above with $O(C(t)) \neq 1$. Since $O(C(t))$ is of odd order it follows that there is a $q$-subgroup $Q \neq 1$ of $O(C(t))$ which is normal in $C(t)$. Thus, the $q$-local subgroup $N(Q)$ contains the entire centralizer $C(t)$. Now we can start to study the $q$-local subgroup using this information. One can go quite far. The aim is to prove, in contradiction to the supposed simplicity of $G$, that $G$ does have a nonidentity normal subgroup of odd order.

This discussion should serve as motivation for the introduction of the next concept. Let $P$ be a nonidentity $p$-subgroup of a group $G$, for example $p=2$ and $P$ a Sylow 2-subgroup of $C(t), t$ an involution. The signalizers for $P$ are the $p^{\prime}$-subgroups-that is, subgroups of order prime to $p$-of $G$ which are normalized by $P$. Thus, if $Q$ is a $q$-subgroup for a prime $q$ other than $p$, then $Q$ is a $P$-signalizer if $P$ is contained in the $q$-local $N(Q)$. The above discussion about the centralizer of an involution leads to the following two general questions: survey all the $P$-signalizers; put various $P$-signalizers together to generate even bigger $P$-signalizers. This leads to two important topics: transitivity theorems; signalizer functors. We shall devote the remainder of the section to exploring these two ideas. 
Let $P$ be a $p$-subgroup of the group $G$ and let's look at the collection of signalizers for $P$ which are $q$-subgroups of $G$ for another prime $q$. If $P$ is just the identity then we are examining the set of all $q$-subgroups of $G$. We then know that there are some remarkable theorems which hold, namely, the Sylow theorems. One way of stating part of these results is the following: the maximal $q$-subgroups of $G$ are all conjugate. Thus, every $q$-subgroup is contained in a maximal $q$-subgroup and all these are conjugate. Now suppose that $P$ is not the identity subgroup so the $q$-subgroups which are signalizers for $P$ will probably be a proper subset of the collection of all $q$-subgroups of $G$. But could there be a version of Sylow's theorem that holds for these signalizers? The answer is that sometimes there is. Such a result is called a transitivity theorem.

Thus, sometimes it is possible to prove that all the $q$-subgroups, maximal subject to being normalized by $P$, are conjugate in $G$. But more is the case. One must consider $P$ as being as a group of operators. Each of the signalizers is invariant under conjugation by elements of $P$. The transitivity results usually prove that the conjugation that occurs between maximal $P$-signalizers commutes with the action of $P$. That is, if $Q_{1}$ and $Q_{2}$ are $q$-subgroups, maximal subject to being $P$-signalizers, then one of ten has that there exists $x$ in $C(P)$ with $Q_{1}$ conjugated to $Q_{2}$ by $x$.

This in turn can be used to get some useful consequences. So let $Q$ be one of these $q$-subgroups, maximal subject to being a $P$-signalizer. If $g$ is an element of the $p$-local subgroup $N(P)$ then $P=g^{-1} P g$ normalizes $Q$ and $g^{-1} Q g$. The latter subgroup is also one of these maximal signalizers, so, if transitivity holds in full, there is $c$ in $C(P)$ such that $c^{-1}\left(g^{-1} Q g\right) c=Q$. Hence, $g c$ is in $N(Q)$ and $g$ is in $N(Q) C(P)$. This implies that

$$
N(P)=C(P)(N(Q) \cap N(P)) .
$$

This says that a lot of $N(P)$, a $p$-local subgroup, is contained in the $q$-local subgroup $N(Q)$. Indeed, suppose that $P$ contains its own centralizer. Then we have $N(P) \subseteq P(N(Q) \cap N(P))$ which is contained in $N(Q)$. The $p$-local subgroup $N(P)$ is contained in the $q$-local subgroup $N(Q)$. We are really starting to get somewhere with this sort of geometry of local subgroups!

Now we turn to signalizer functors. We let $A$ be an elementary abelian $p$-subgroup of some group $G$, not necessarily simple. (Thus, $A$ is abelian, a $p$-subgroup and is of exponent $p$.) An $A$-signalizer functor on $G$ is a function $\theta$ from the nonidentity elements of $A$ to the subgroups of $G$ which satisfies the following conditions:

1. $\theta(a)$ is a $p^{\prime}$-subgroup of $C(a)$ normalized by $A$, for each nonidentity element $a$ of $A$;

2. If $a$ and $b$ are nonidentity elements of $A$ then $C(b) \cap \theta(a) \subseteq \theta(b)$.

Let's give an example of such a functor. Let $N$ be a normal subgroup of the group $G$ and assume that $N$ has order prime to $p$. Let $A$ be as above and define 


$$
\delta(a)=C(a) \cap N
$$

for each nonidentity element $a$ of $A$. Then $\delta$ is a signalizer functor. Indeed, $N$ and $C(a)$ are both normalized by $a$ so that so is their intersection. And this intersection is also a $p^{\prime}$-subgroup since it is contained in the $p^{\prime}$-subgroup $N$. Hence, the first condition holds. As for the second, we simply calculate very easily,

$$
\begin{aligned}
C(b) \cap \delta(a) & =C(b) \cap(C(a) \cap N) \\
& \subseteq C(b) \cap N=\delta(b) .
\end{aligned}
$$

Could this be the typical signalizer functor? That is, if $\theta$ is an $A$-signalizer functor on $G$ could there have to be a normal $p^{\prime}$-subgroup $N$ with $\theta(a)=$ $C(a) \cap N$ ? Well, not always, but usually! This is really surprising because the hypotheses are so minimal. But the second axiom is really much stronger than it looks.

5. The $B$-conjecture. In the preceding discussion of centralizers of involutions in simple groups we saw that one often aimed at statements like the following: $O(C(t))=1$. In general, if $G$ is any group and $L$ is a $p$-local subgroup of $G$ one would like to have that

$$
O_{p^{\prime}}(L) \subseteq O_{p^{\prime}}(G),
$$

where these denote the largest normal $p^{\prime}$-subgroups of $L$ and $G$, respectively. For example, if $p=2$ and $G$ is simple then this would imply that $O(L)=1$ and $L$ might be the centralizer of an involution. This is not true in general but there is a weakened form of this statement which is conjectured to hold always:

$$
O_{p^{\prime}}\left(B_{p}(L)\right) \subseteq O_{p^{\prime}}\left(B_{p}(G)\right) .
$$

Here $B_{p}$ assigns to every finite group a characteristic subgroup, so the two sides of the containment are contained in $O_{p^{\prime}}(L)$ and $O_{p^{\prime}}(G)$, respectively. In particular, if $G$ is simple and of order divisible by $p$ then the truth of this conjecture would imply that $O_{p^{\prime}}\left(B_{p}(L)\right)=1$.

The definition of $B_{p}$ is technical so let's state this conjecture in another form which is easier to understand. To do this we need to introduce another subgroup. If $H$ is any group there is a largest normal subgroup $E(H)$ with the following properties:

1. $E(H) / Z(H)$, the quotient of $E(H)$ by its center, is the direct product of nonabelian simple groups;

2. $E(H)$ has no nonidentity abelian quotient group.

That such a subgroup exists is a point we shall return to at the end of this section. Now, if $L$ is any $p$-local subgroup of $G$ let $\bar{L}$ denote the quotient $L / O_{p^{\prime}}(L)$ and for any subset $X$ of $L$ let $\bar{X}$ be the image of $X$ in $\bar{L}$. The 
$B$-conjecture is then equivalent to the following assertion: If $O_{p^{\prime}}(G)=1$ then $E(\bar{L})=\overline{E(L)}$.

It looks so innocent. But a direct proof would be of very great importance. The case $p=2$, which is the most important case, is a subject of tremendous activity. A whole body of group theory is being created to prove this conjecture for $p=2$. But one still wonders is there any way of proving this result directly. Since it holds for all groups and all primes one can guess that block theory is relevant. It is true that the classification of simple groups-provided nothing unpleasant turns up-would resolve the conjecture. But its purpose is to be a fundamental step in that classification so that would be a very hollow victory over this conjecture.

The rest of this section is devoted to sketching how one proves the existence and properties of the subgroup $E(H)$ and related subgroups which are now so important. Since we shall be asking more of the readers, some of them may wish to skip ahead to our section of conclusions.

Our aim now is to define $B_{p}$ but we shall first have to disucss $E$ and other such operators. Recall that a group $H$ is nilpotent if it satisfies the following equivalent conditions: $H$ centralizes each of its chief factors; $H$ is the direct product of its Sylow subgroups. Every group $G$ has a largest normal nilpotent subgroup called the Fitting subgroup and denoted $F(G)$. For our purposes here only, we call a group mock nilpotent if it induces only inner automorphisms on its chief factors. It is now true that every group $G$ possesses a largest normal mock nilpotent subgroup called the generalized Fitting subgroup and denoted $F^{*}(G)$.

Let's see why this is so. First, quotients and direct products of mock nilpotent groups are again mock nilpotent. Thus, if $N_{1}$ and $N_{2}$ are normal mock nilpotent subgroups of $G$ then so is $N_{1} N_{2} / N_{1} \cap N_{2}$ since it is isomorphic with $N_{1} / N_{1} \cap N_{2} \times N_{2} / N_{1} \cap N_{2}$. Furthermore, if $K / L$ is a chief factor of $N_{1} N_{2}$ below $N_{1} \cap N_{2}$ then it also has only inner automorphisms induced by $N_{1} N_{2}$. Indeed, since $N_{1}$ is a normal subgroup of $N_{1} N_{2}, K / L$ is a direct product of chief factors of $N_{1}$. Each of these has only inner automorphisms induced by $N_{1}$ so the same is true of $K / L$. A similar argument applies to $N_{2}$. Hence, $N_{1} N_{2}$ is mock nilpotent and so the product of all the mock nilpotent normal subgroups is again such a subgroup and $F^{*}(G)$ is as stated.

A critical property of $F^{*}(G)$ is that it contains its own centralizer in $G$. Indeed, suppose that $C$ is this centralizer and that $C$ properly contains $C \cap F^{*}(G)=Z\left(F^{*}(G)\right)$. If $K / Z\left(F^{*}(G)\right)$ is a chief factor of $K$ then it is a direct product of simple groups. Furthermore, $K$ centralizes $Z\left(F^{*}(G)\right)$ so $K$ is a normal mock nilpotent subgroup not contained in $F^{*}(G)$, a contradiction.

Now we turn to the structure of $F^{*}(G)$. First, $F^{*}(G)$ contains $F(G)$ since $F(G)$ is even nilpotent. We assert that the quotient $F^{*}(G) / F(G)$ is semisimple, that is, a direct product of nonabelian simple groups. Indeed suppose that $K / F(G)$ is the Fitting subgroup of $F^{*}(G) / F(G)$. Thus, $K$ is certainly 
solvable. But $K$ is normal in $F^{*}(G)$ so it must be mock nilpotent as each of the chief factors of $F^{*}(G)$ below $K$ is the direct product of chief factors of $K$. Hence, $K$ is even nilpotent so we must have $K=F(G)$. Thus, our assertion will hold once we prove the following: if $H$ is a mock nilpotent group and $F(H)=1$ then $H$ is semisimple. Let $S$ be the socle of $H$ so $S$ is semisimple as $H$ has no normal abelian subgroups. Since $H$ is mock nilpotent we have that $H=S C(S)$. Since $S$ is semisimple we have $S \cap C(S)=1$. But $C(S)$ is normal in $H$ so if were a nonidentity subgroup it would intersect the socle $S$ in a nonidentity subgroup. Thus, $C(S)=1$ and $H$ is as stated. Moreover, the semisimplicity of $F^{*}(G) / F(\mathrm{G})$ is also established.

Letting $C$ be the centralizer of $F(G)$ in $F^{*}(G)$ we now claim that $F^{*}(G)=$ $F(G) C$. Indeed, $F^{*}(G) / C$ is a group of automorphisms of $F(G)$ and since $F^{*}(G)$ is mock nilpotent it stabilizes a series of normal subgroups of $F(G)$. Therefore, $F^{*}(G) / C$ is nilpotent and so $F^{*}(G) / F(G) C$ is also nilpotent. But $F^{*}(G) / F(G)$ is semisimple and its only nilpotent quotient is the identity. Thus, $F^{*}(G) / F(G) C=1$ and our claim holds.

Since $F^{*}(G) / F(G)$ is semisimple so is $C / C \cap F^{*}(G)$. Thus, if we let $E(G)$ be the terminal member of the derived series of $C$ (and so $E(G)=C^{\prime}$ in fact) then we have immediately the following: $F^{*}(G)=F(G) E(G)$; $E(G)=E(G)^{\prime} ; E(G) / Z(E(G))$ is semisimple; $E(G)$ is a characteristic subgroup of $G$. Moreover, suppose that $H$ is a normal subgroup of $G$, that $H=H^{\prime}$ and $H / Z(H)$ is semisimple. In particular, $H$ is mock nilpotent. Thus, $H$ is contained in $F^{*}(G)$ and $H$ is even contained in the terminal member of the derived series of $F^{*}(G)$ which is $E(G)$ since $F(G)$ is certainly solvable.

An easy induction shows that if $H$ is subnormal and has these other properties then $H$ is still contained in $E(H)$. In particular, if $H$ is a component of $G$, that is, subnormal, $H=H^{\prime}$ and $H / Z(H)$ is a nonabelian simple group, then $H$ is contained in $E(H)$. In fact, it is easy to see that if we express $E(H) / Z(E(H))$ as the direct product of simple groups $S_{1} / Z(E(H)), \ldots, S_{n} / Z(E(H))$ then the components of $G$ are exactly the subgroups $S_{1}^{\prime}, \ldots, S_{n}^{\prime}$.

We shall now relativize these concepts with respect to the prime $p$. We let $E_{p}(G)$ be the subgroup of $G$ containing $O_{p^{\prime}}(G)$ such that $E_{p}(G) / O_{p^{\prime}}(G)=$ $E\left(G / O_{p^{\prime}}(G)\right)$. It follows that the center of $E\left(G / O_{p^{\prime}}(G)\right)$ is a $p$-group and the quotient by that center is a direct product of nonabelian simple groups, each of order divisible by $p$. The $p$-layer of $G$, denoted by $L_{p}(G)$, is then defined by

$$
L_{p}(G)=O^{p^{\prime}}\left(E_{p}(G)\right),
$$

the smallest normal subgroup of $E_{p}(G)$ whose quotient is a $p^{\prime}$-group. The structure of $E_{p}(G) / O_{p^{\prime}}(G)$ yields that

$$
L_{p}(G) O_{p^{\prime}}(G)=E_{p}(G) \text { and } L_{p}(G) \cap O_{p^{\prime}}(G)=O_{p^{\prime}}\left(L_{p}(G)\right) .
$$

This can be described by a picture: 


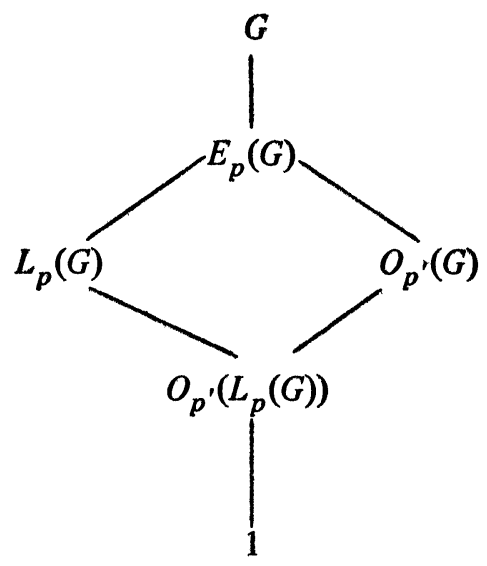

The subgroup $L_{p}(G)$ is the important one. We could also have reached it by using the concept of $p$-nilpotence and introducing mock $p$-nilpotent groups. With these ideas $L_{p}(G)$ becomes the relativized version of $E(G)$.

Just as we have components we also have $p$-components. A subgroup $H$ of $\boldsymbol{G}$ is a $p$-component provided it satisfies the following conditions: $H$ is subnormal; $H=H^{\prime} ; H=O^{p^{\prime}}(H)$; if $K=H / O_{p^{\prime}}(H)$ then $K / Z(K)$ is a nonabelian simple group. It then follows that $L_{p}(G)$ is the product of all the $p$-components of $G$. In fact, these $p$-components correspond one-to-one with the simple direct factors of the central quotient group of $L_{p}(G) / O_{p^{\prime}}\left(L_{p}(G)\right)$.

At long last we're ready to define $B_{p}(G)$. It is simply the product of all the $p$-components of $G$ which are not actually components. The celebrated $B$-conjecture simply states that for any $p$-local subgroup $M$ of a group $G$ we have that $O_{p^{\prime}}\left(B_{p}(M)\right)$ is contained in $O_{p^{\prime}}\left(B_{p}(G)\right)$.

6. Conclusions. We've certainly been over a lot of ground; let's briefly recapitulate. We started with the notion of a local subgroup and with some examples to show that the interesting subgroups-at least a lot of them-are among the local subgroups. We've followed this up with a survey of some of the highlights of local group theory. First, and unfortunately only very briefly, we looked at the structure of a single $p$-local subgroup. We did this only as far as to see where the idea of a module comes in. That point of view is really fundamental. The next two sections dealt with the geometry-if you like-of the local subgroups. First, we looked at the case of all the $p$-local subgroups of a group and only touched on the vast area of how one moves from one $p$-local subgroup to another. But we hopefully gave the reader some idea of how the $p$-local structure of a group really does tell one a lot about the group. This was followed up by our discussion of the semilocal theory at least as far as the machinery that could be formalized neatly. There are other topics that could be described there too.

In any case, we hope that you have some idea of how a group can be built up doing local analysis. And perhaps some feeling for how natural and right this is. And even how inevitable. In particular, the formality of a number of 
the results-the local to global machinery, signalizers and transitivity, signalizer functors-is some evidence that a lot of the basic ideas have surfaced.

Finally, perhaps there are some lessons about the nature of mathematics to be learned here. It's possible to piece together a structure bit by bit patiently doing one thing and another and moving about and finally reaching the answer even after a great number of pages of mathematics have been written. There are other places where this philosophy can be applied.

Department of Mathematics, University of Chicago, Chicago, Illinois 60637 